\title{
Brain pathology in Adult Rats Treated with Tetrodotoxin
}

\author{
Thilagar Gobinath, Samuthirapandian Ravichandran* \\ Centre of Advanced Study in Marine Biology, Faculty of Marine Sciences, Annamalai University, Parangipettai - 608 502, Tamil Nadu, India
}

\author{
DOI: $10.36348 /$ sjpm.2019.v04i11.014 \\ | Received: 18.11.2019| Accepted: 25.11.2019| Published: 28.11.2019
}

*Corresponding author: Samuthirapandian Ravichandran

\section{Abstract}

Puffer fish poisoning remains a severe seafood poisoning condition blamable for numerous human intoxications. Research on puffer fish poisoning enables studying the involvement of puffer fish poisoning in human security. The current study thus intention of investigating whether Arothron hispidus from the south-east coast of India (Palazhyar) cause for the neurodegeneration. Cresyl violet stained sections of the rat brain regions examined for pathological changes in rats treated with A.hispidus toxic extract. We noticed disarrangement and neuron loss in the primary motor area, hippocampus, amygdala, thalamus, temporal lobe, parietal lobe, and cerebellum regions of rats treated with A.hispidus skin extract. Damaged regions directly or secondarily associated with sensory and motor functions, this may cause loss of sensory and motor function. From our observation, we conclude that neuronal loss of a rat's brain treated with skin extract as clear through histopathological analysis, due to the neurotoxic effect of Arothron hispidus.

Keyword: Arothron hispidus; neurotoxic; poisoning; sensory functions; motor functions; neuron loss.

Copyright @ 2019: This is an open-access article distributed under the terms of the Creative Commons Attribution license which permits unrestricted use, distribution, and reproduction in any medium for non-commercial use (NonCommercial, or CC-BY-NC) provided the original author and sources are credited.

\section{INTRODUCTION}

Tetrodotoxin (TTX) is a naturally occurring potent neurotoxin produced by the marine puffer fish, bacteria and gobies, newts, frogs, horseshoe crabs, xanthid crabs, blue-ringed octopus, gastropods, starfish, flatworms, ribbon worms, annelids, arrow worms, red calcareous alga, and dinoflagellates, etc [1]. Once transferee to the humans, TTX a guanidinium toxins acts to block the Voltage Gated Sodium Channels (VGSCs) in the neurons and skeleton muscles [2]. TTX poisoning is majorly caused by ingestion of puffer fish when mistakenly eaten strong toxin parts such as liver, ovary, etc. Several tens of peoples are poisoned annually and 2 to 3 were killed as a result of puffer fish poisoning [2]. The affected peoples presented numbness, tingling, salivation, nausea, and vomiting. In severe cases might take place with respiratory problems, cardiac failure, and death [3].

The best way to determine the toxicity of TTX isolated from puffer fish is use rodent models. A recent acute oral study with mice established that the $\mathrm{LD}_{50}$ is $232 \mu \mathrm{g} \mathrm{TTX} / \mathrm{kg} \mathrm{BW}$ and that $25 \mu \mathrm{g} / \mathrm{kg} \mathrm{BW}$ had non observed adverse effects [1]. Several previous studies demonstrated the pathological effects of TTX of certain regions of brain such hippocampus. The goal of the present study was to contribute to the knowledge on the oral acute toxic effects of TTX isolated from A.hispidus in a rat model with a particular focus on the microscopic alterations in Primary motor area, Hippocampus (CA1, CA2, CA3 and DG) Amygdala, thalamus, neocortex and cerebellum.

\section{MATERIALS AND METHODS \\ Animals Purchase and Housing}

Male albino wistar rats weighed around 150 grams purchased from the Biogen animal breeding facility (Bangalore, India). The rats fed on a standard pellet diet. All studies were conducted under the National Institutes of Health Guide for the Care and Use of Laboratory Animals, and the study was approved by the Ethical Committee of Rajah Muthiah College and Hospital (AU-IEAC/1186/1/18), Annamalai University, Annamalai Nagar.

\section{Fish Collection and Extract Preparations}

Puffer fish A.hispidus specimens collected from Palazhyar $\left(11.3494^{\circ} \mathrm{N}, \quad 79.8171^{\circ} \mathrm{E}\right)$ in Nagapattinam (south-east coast of India). Immediately after collection fishes were frozen and transported to the lab, in clean lab condition fishes were dissected and organs (liver, skin, and muscles) were taken out. $1 \mathrm{gm}$ of tissue from each organ homogenized with $4 \mathrm{ml}$ of 0.1 $\%$ of the acetic acid solution, and boil the extract at $80^{\circ} \mathrm{C}$ for 10 minutes and allowed to cool up to room temperature. The extract was centrifuged (10,000 RPM) for $20 \mathrm{mins}$ and the supernatant stored at $-20^{\circ} \mathrm{C}$. 


\section{Assay of Toxicity}

Toxicity of tissues measured by mouse bioassay, toxicity in a mouse bioassay expressed as mouse unit (MU), and one mouse unit is defined as the amount of toxin that enough to kills a mouse in 30mins after Intraperitoneal injection [4].

\section{LC-MS Analysis}

The $0.1 \%$ acetic acid extract of skin was ultrafiltered through a membrane (limit of 10,000 Da), and submitted to LC/MS analysis on an alliance LC/MS system equipped with an MS 2000 detector [5]. As LC conditions, a Puresil C18 (4.6250 mm, Waters) column was used with $30 \mathrm{mM}$ heptafluorobutyric acid in $1 \mathrm{mM}$ ammonium acetate buffer ( $\mathrm{pH}$ 5.0) for a mobile phase, and the flow rate was set at $1.0 \mathrm{ml} / \mathrm{min}$. About $20 \%$ of the eluate was introduced via a splitter into the ion source of MS, and ionized by a positive mode of electrospray ionization (ESI) with desolvation temperature at $350^{\circ} \mathrm{C}$, source block temperature at $120^{\circ} \mathrm{C}$, and cone voltage at $30 \mathrm{~V}$.

\section{Experimental Design}

After acclimatization for 10 days rats were divided into four groups which were daily orally administered with, $1 \mathrm{ml}$ of physiological saline/100 gms of the body weight for Group $-1.75 \mu \mathrm{g} / \mathrm{kg}$ of skin extract for Group-2. $125 \mu \mathrm{g} / \mathrm{kg}$ of skin extract for the Group-3 and $250 \mu \mathrm{g} / \mathrm{kg}$ of skin extract for Group - 4 for 15 days.

\section{Tissue Collection and Fixation}

After completion of experiment rats were euthanized with ketamine and perfused intracardially with physiological saline for 2 minutes, followed by 10 $\%$ of neutral buffered formalin for fixation of tissues for ten minutes. The brain was dissected out carefully and kept in an ice-cold platter and washed with saline solution followed by tissues were stored at $10 \%$ neutral buffered formalin.

\section{Cresyl Violet (CV) Staining}

Tissue sections were stained with $\mathrm{CV}$ to evaluate the morphological changes. Deparaffinized and rehydrated sections were washed with distilled water and stained with CV for 1 hour, and slides were washed with distilled water and dried overnight and preserved using DPX mountant. The CV-stained sections observed under a light microscope (10 and 40X) and photographs were taken for further investigations.

\section{RESULTS \\ Mouse Bioassay}

Mouse bioassay was carried to analyze the toxicity effects of TTX isolated from A.hispidus, but in the present study no mortality was recorded. However typical symptoms like restless motion, excessive thirst, shivering of the limps and partial paralysis were recorded. These symptoms are mostly recorded in the animals treated with $A$. hispidus skin extract.

\section{LC-MS analysis of puffer fish toxin}

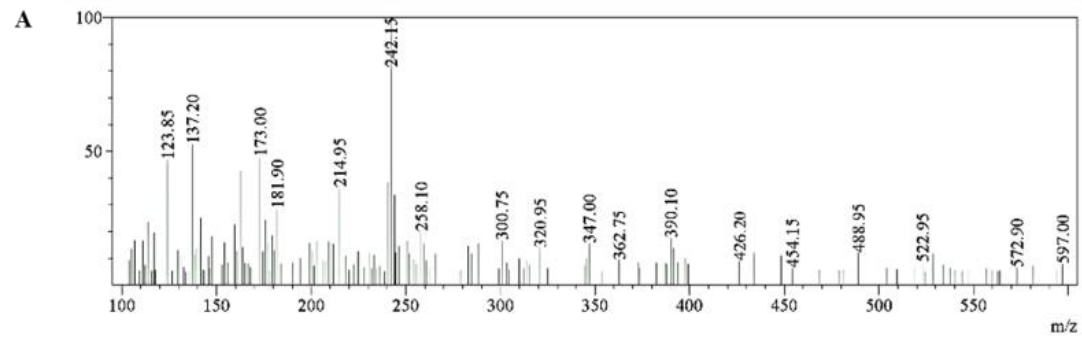

B

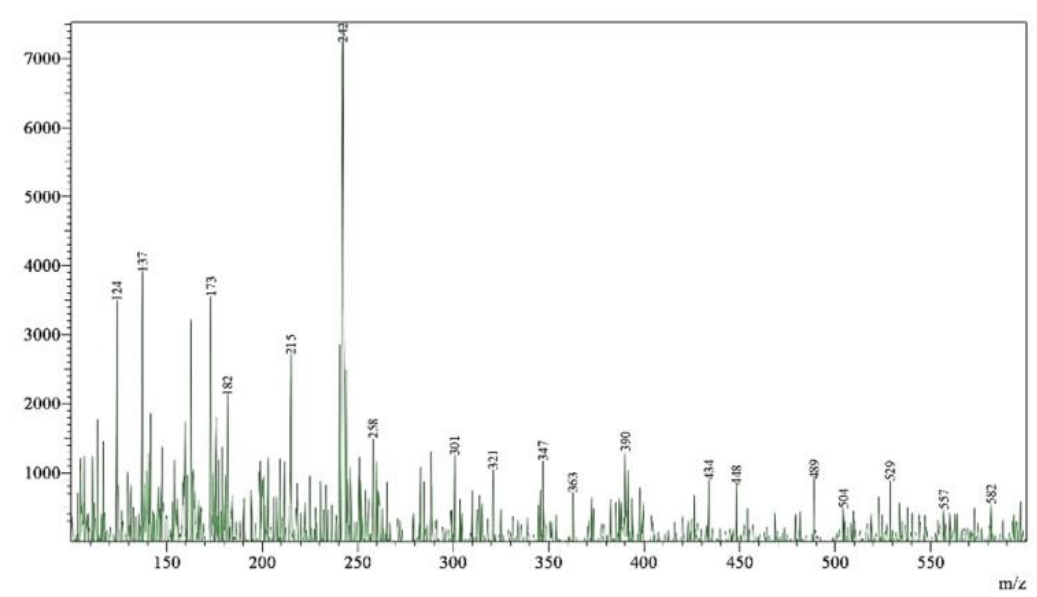

Fig-1: (A) Mass spectrogram of the skin extract from A.hispidus, (B) MS spectra of $\mathbf{m} / \mathbf{z} 320$ of the skin extract from A. hispidus 
In order to confirm that the skin extract from A. hispidus contained truly TTX, LC/MS was used. The mass spectrogram (Fig-1A) shows that the peak from $A$. hispidus occurs at $\mathrm{m} / \mathrm{z} 320$ corresponding to the pronated molecular ion $(\mathrm{M}+\mathrm{H})^{+}$as TTX has a molecular mass of 319. AnhydroTTX and deoxyTTX were also detected in the extract (data not shown). The fragmentation products of TTX peak $(\mathrm{m} / \mathrm{z} 320)$ in skin of $A$. hispidus after being subjected to MS are shown in Fig-1B.

\section{Structural Examination \\ Primary Motor Area}

$\mathrm{CV}$-stained sections of the primary motor area examined microscopically, animals administered with $125,250 \mu \mathrm{g} / \mathrm{kg}$ show extensive neuronal loss throughout the structure (Fig.2). Contracted, vacuolated and densely stained neurons were identified through the primary motor area layers 1, 2/3, 5, 6a, 6b. Rats did not present any evidence of primary motor area damage with the lowest dose of $75 \mu \mathrm{g} / \mathrm{kg}$.

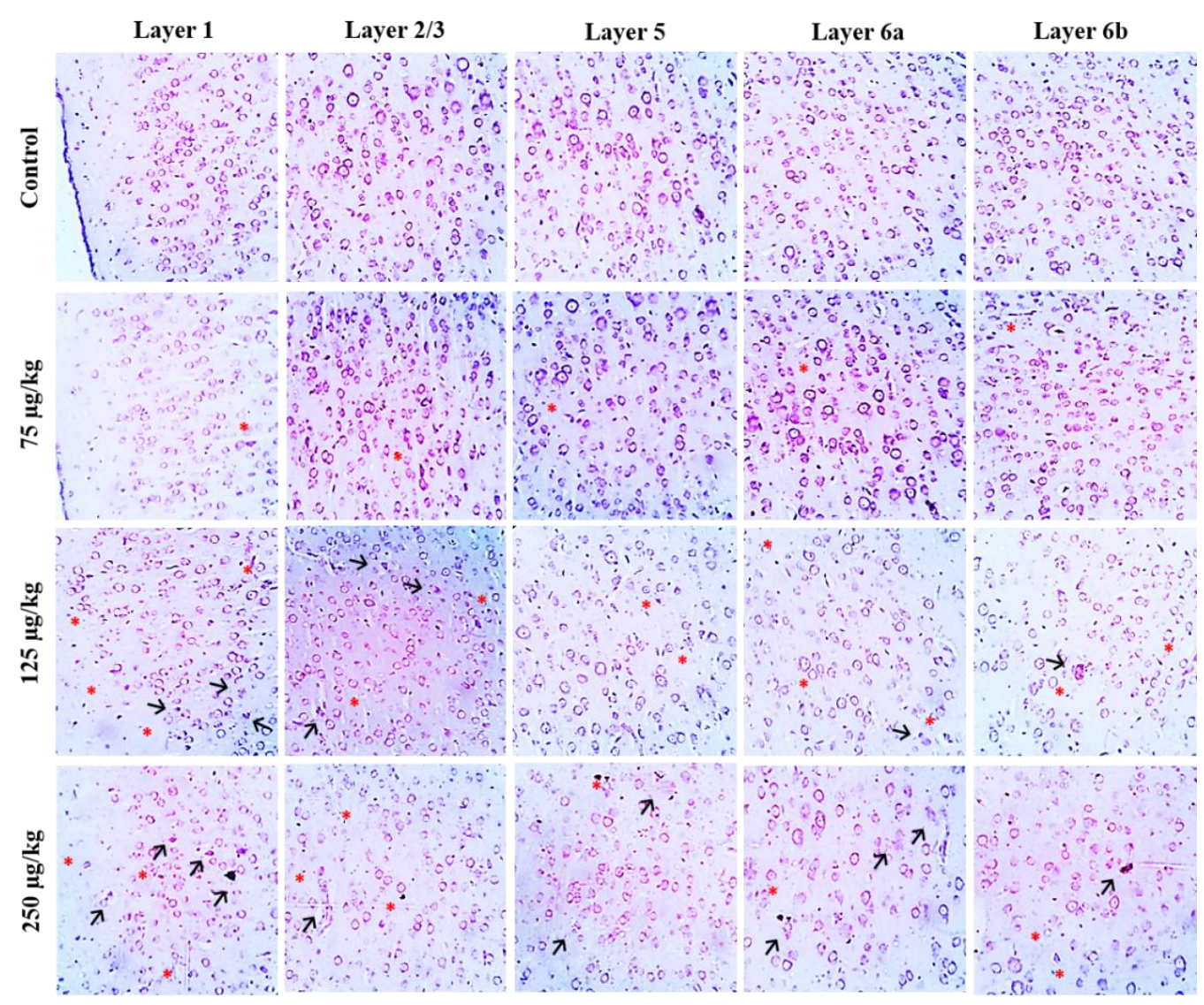

Fig-2: Picture of nissl stained primary motor cortex (coronal section). High magnification (40X) images of layer 1, Layer 2/3, (C) Layer 5, Layer 6a, and Layer 6b. Dead cell (asterisk) and Dark neurons (arrow)

\section{Hippocampus}

In DG the insignificant level of neuronal misalignment in granule cell layer was evident in animals treated with 125 and $250 \mu \mathrm{g} / \mathrm{kg}, 75 \mu \mathrm{g} / \mathrm{kg}$ does not show any alterations when compared with control (Fig-3). In addition, we continued our investigation on other regions of the hippocampus (CA1, CA2, and CA3) to clarify the effects of $A$. hispidus toxin on other regions of the hippocampus. No neuronal alterations observed in the hippocampus from the animals of a control group. Light microscopic observations revealed that no neuronal modifications in CA1 region of experimental animals (Fig-3). Qualitative observations revealed neuronal loss in $\mathrm{CA} 2$ and $\mathrm{CA} 3$ regions of the experimental animals, specifically in 125 and $250 \mu \mathrm{g} / \mathrm{kg}$ treated animals. 125 and $250 \mu \mathrm{g} / \mathrm{kg}$ treated groups evidenced with loss thickness of pyramidal cell layer, a lethal level of neuronal loss, vacuolation, misalignment of pyramidal cells. 


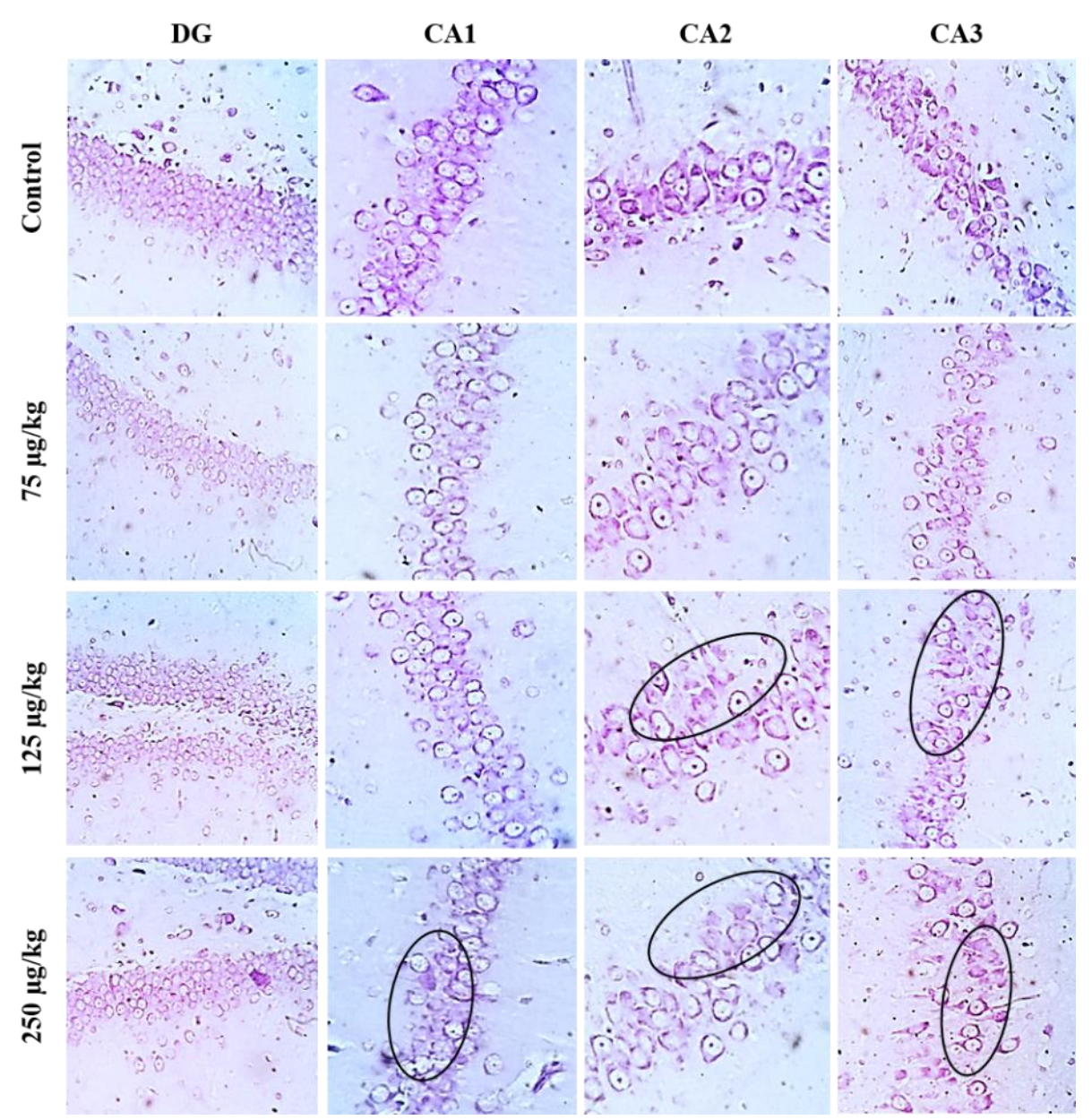

Fig-3: Effect of puffer fish skin extract in various subregions of the hippocampus of the experimental rats. Dentate Gyrus (DG), Cornu ammonis 1 (CA1), Cornu ammonis 2 (CA2), Cornu ammonis 3 (CA3). The pattern of the pyramidal cells in the hippocampus showed disrupted and misalignment of DG, CA1, CA2 and CA3 in TTX treated rats. Disrupted pattern were clearly visible in 125 and $250 \mu \mathrm{g} / \mathrm{kg}$ experimental rats (encircled)

\section{Amygdala and Thalamus}

It is indistinguishable that pathological changes in hippocampus regions associated with other regions of the brain associated with motor function, to clarify the doubt we examined the next suspected regions. Histopathological analysis of amygdala in the control and experimental groups of the rat brain shown in Fig.4A. CV-stained sections showed a normal structure with clear, intact neurons in the amygdala region of the control rat brain.
However, 125 and $250 \mu \mathrm{g} / \mathrm{kg}$ treated rats showed pathological alterations such as neuronal loss and dead cells when compared with corresponding control. Whereas, no specific abnormalities observed between $75 \mu \mathrm{g} / \mathrm{kg}$ treated rats. Changes in the amygdala may also affect the thalamus connections, in the current study neuronal damage was evidenced throughout the thalamus of $250 \mu \mathrm{g} / \mathrm{kg}$ treated animals followed by 125 $\mu \mathrm{g} / \mathrm{kg}$ (Fig.4B). Neuronal damage, loss of neurons, shrunken cells observed in the thalamus of $250 \mu \mathrm{g} / \mathrm{kg}$ treated animals as compared to control animals. 


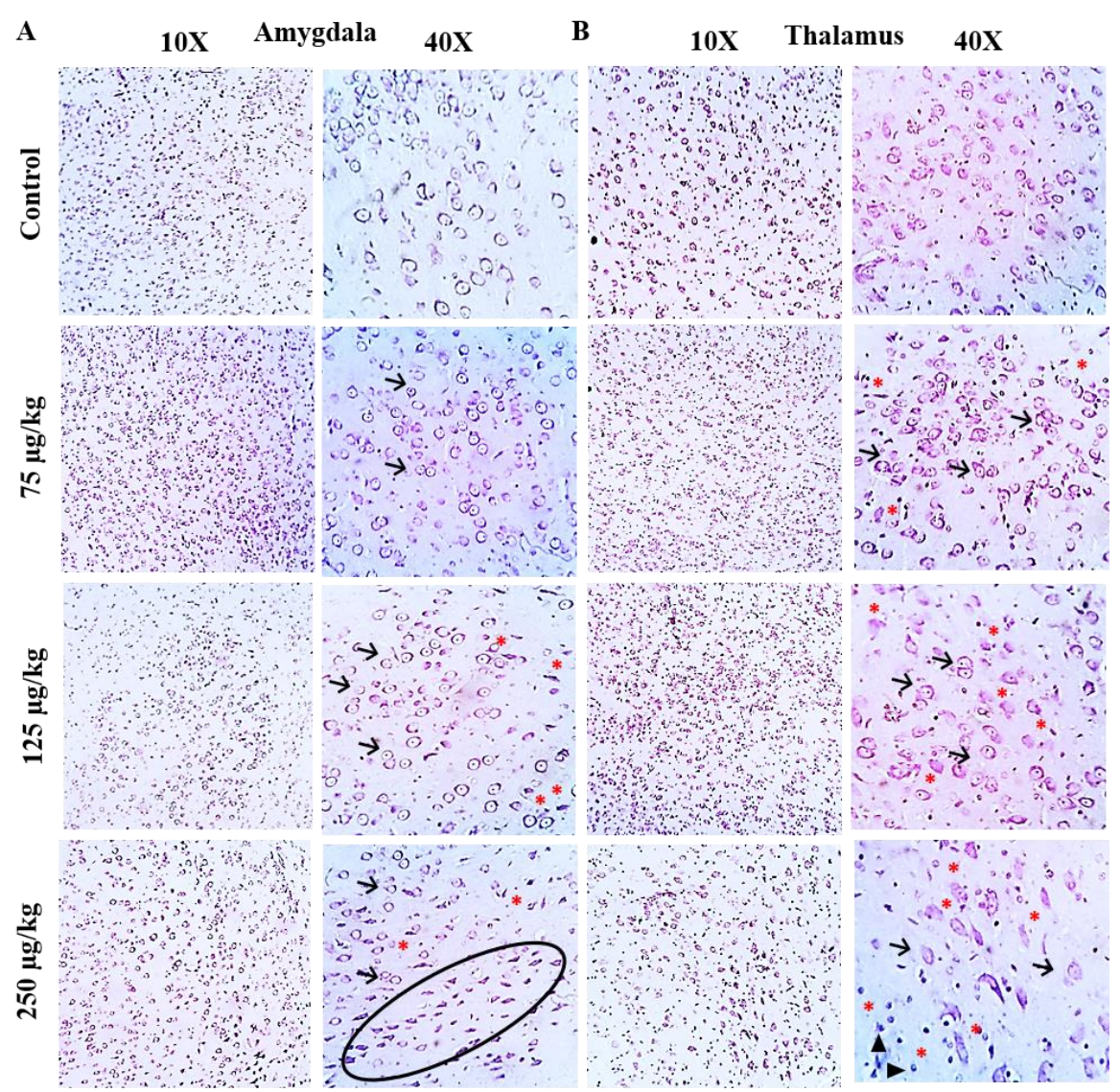

Fig-4: Effect of TTX on Histopathological changes in the (A) amygdala and (B) thalamus. Low-magnification (10) and high-magnification (40) images at coronal sections of the amygdala and thalamus showing intact neurons (arrow, clear rounded cells having a distinct nucleus), Glial cells (arrowhead) and dead cells (asterisk)

\section{Neocortex}

The present study evidenced that primary motor area along with adjacent regions associated with sensory and motor functions were damaged during $A$. hispidus poisoning. However, it remained a mystery, whether degeneration of neurons in the layers of the neocortex is normal during A. hispidus poisoning. To clarify this question, six-layered structure of both temporal and parietal cortex of neocortex regions investigated for pathological changes during A. hispidus poisoning. CV stained pathological analysis of neocortex regions temporal revealed that there is an increased level of neuron loss in specifically in layer (I, II and III) (Fig-5B) of the temporal lobe. In temporal lobe specifically in layer I, II and III show neuronal loss, cell shrunken, and dark stained neurons were evident in animals treated with $250 \mu \mathrm{g} / \mathrm{kg}$. Interestingly, we cannot observe any pathological changes in layer IV, $\mathrm{V}$ and VI of the temporal lobe. However, $75 \mu \mathrm{g} / \mathrm{kg}$ do not show any alterations in all layers as compared with respective control. Additionally, parietal lobe well analyzed for pathological alterations through control and treated animals (Fig-5A). No pathological changes observed in the parietal lobe from the control and $75 \mu \mathrm{g} / \mathrm{kg}$ treated group. Histopathological analysis revealed that dead cells, neuronal loss, and vacuolation in layer I, II and III of parietal lobe evidenced from 250 $\mu \mathrm{g} / \mathrm{kg}$ animals as compared with a control group. No neuronal modifications evidenced in parietal lobe layer $\mathrm{IV}, \mathrm{V}$, and VI of $250 \mu \mathrm{g} / \mathrm{kg}$ treated animals. Fascinatingly, the analysis revealed that no pathological alterations in rats treated with 125 and $75 \mu \mathrm{g} / \mathrm{kg}$ when compared with control. 

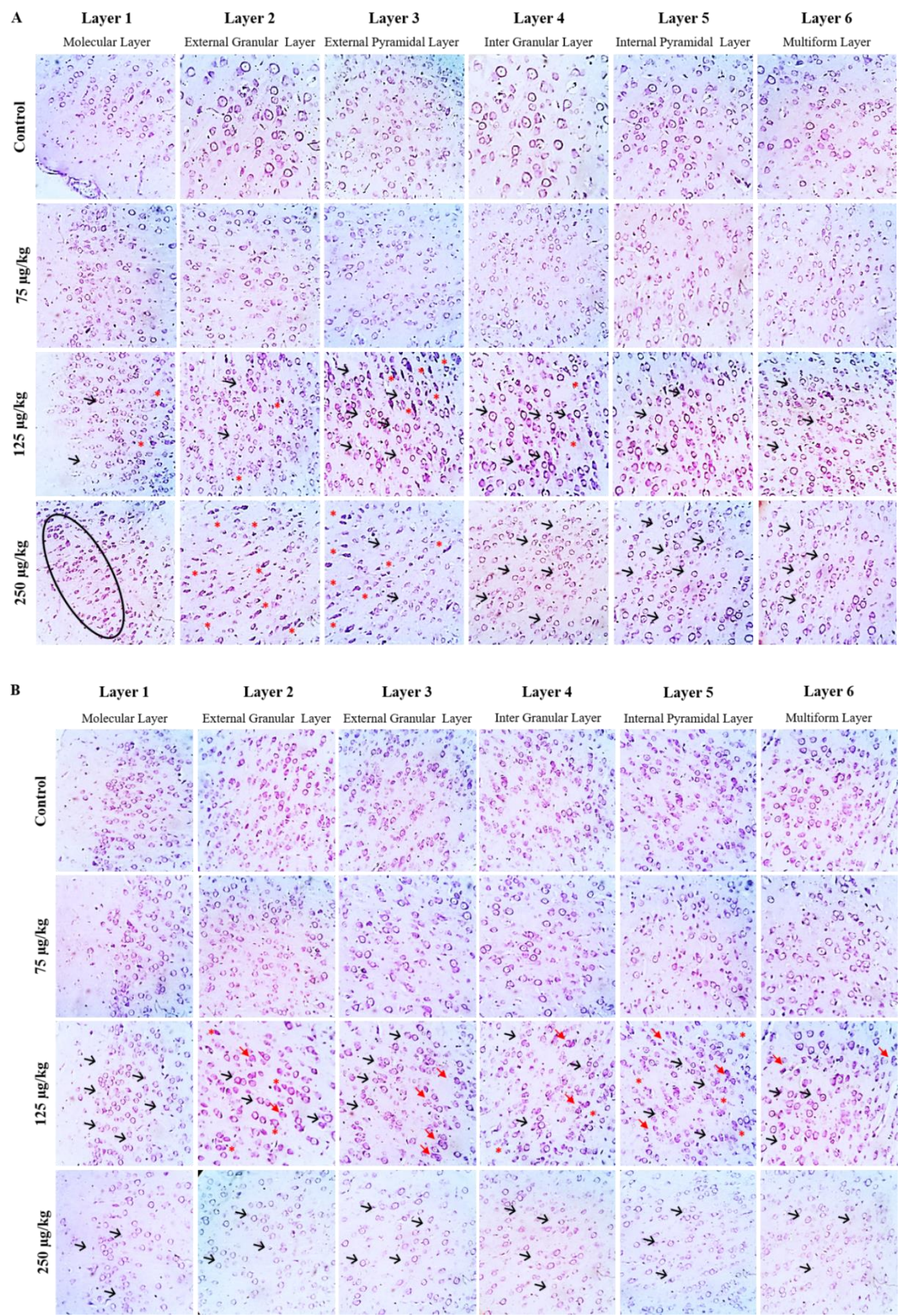

Fig-5: Effect of TTX in various subregions of the neocortex of the experimental rats. (A) Parietal lobe (B) Temporal lobe. High neuronal death (encircled), dark neurons (red arrow), and dead cell (asterisk) were observed in the 125 and $250 \mu \mathrm{g} / \mathrm{kg}$ of TTX treated experimental rats Parietal lobe. Whereas Decreased number of surviving neurons (marked with an arrow, clear rounded cells having a distinct nucleus), dark neurons (red arrow), and dead cell (asterisk) were observed in 125 and $250 \mu \mathrm{g} / \mathrm{kg}$ of TTX treated experimental rats Temporal lobe

\section{Cerebellum}

CV stained brain sections show neuronal loss throughout the cerebellum structure when compared with respective control: neuronal loss, apoptotic cells, densely stained neurons identified in 125 and $250 \mu \mathrm{g} / \mathrm{kg}$ treated animals. $75 \mu \mathrm{g} / \mathrm{kg}$ didn't produce any pathological alteration in cerebellum when compared with control animals. 


\section{DISCUSSION}

TTX is a natural poisonous substance derived from puffer fish of the family Tetraodontidae, TTX is the powerful neurotoxin, TTX has caused a 30-50 victims of intoxications within the human around the world once a year [6]. The severity of the symptoms evoked by the TTX is dose-dependent [7]. Current study could use to understand in vivo TTX neurotoxic action in the primary motor area, thalamus, amygdala, hippocampus, neocortex and cerebellum and this could explain whether multiple brain regions generate the majority symptoms as a result of poisoning or the localized effects on some areas of brain such as primary motor area, cerebellum.

Investigation on animal models suggested that puffer fish intoxication produces neuronal cell death in the brain [8]. Similarly, in the current investigation experimentally induced puffer fish poisoning damages are observed in several parts of the brain. Similar type of reaction is parallel to the neuronal alteration seen in patients who had eaten puffer fish [9]. The critical finding of the current study is the neuronal loss of regions associated with sensory and motor functions following puffer fish poisoning. Neuronal damages are more likely the same in the all intoxication induced animals, and the distribution of the damaged area can divide into many sensory and motor functionally connected regions. For example, neuronal degeneration is seen frequently in the primary motor area and hippocampus which are interconnected through motor functions [10].

Volume of neuronal degeneration diverse between the groups and for the most part depends on the dose of poisoning. Regions directly linked with sensory and motor functions are severely damaged, and the degree of neuronal degeneration is still dose depended[9]. In this case, our results indicate that primary motor area, hippocampus DG, CA2, CA3, amygdala, thalamus, and cerebellum is more damaged after puffer fish poisoning. Likewise, damages in those regions suggested that neuronal degeneration in primary motor area and similar degeneration in other areas are not dependable with a simple poisoning condition but instead proposes that sensory and motor neurons more exposed to the possibility for neuronal degeneration [11] and diverse in the neuronal degeneration might be explained by the dose-related toxic action following administration of TTX. Primary motor area, hippocampus DG, CA2, CA3, amygdala, thalamus, and cerebellum becomes direct targets of the puffer fish poisoning due their sensory-perceptual and motor functions $[10,12,13]$.

In Puffer fish poisoning, among the areas more damaged region are the primary motor area where the region of the brain was highly involved in the generation of neural impulse that controls the execution of movement [2]. Damage in the primary motor area or to the downstream connections would cause an immediate weakness of the muscles of the body and face [14]. In the present study the hippocampus is affected later than the primary motor area. The hippocampus plays a key role in the detection of novelty by comparing the incoming stimuli to memories of similar stimuli. Since, hippocampus is involved in motor functions [10]. Pathological changes in DG may have trouble in receiving signals from entorhinal cortex to $\mathrm{CA} 3$, as changes in DG defect in the formation of hippocampus circuit. Damages in CA3 may cause defects in memory formation and fails to process the information from cortex regions that may cause defects in a finding of new stimuli from old situations [10]. It is clear that pathological changes in the hippocampus may act as key players for puffer fish poisoning associated motor function loss.

Amygdala generally involved in both motor and sensory functions [12]. In particular, amygdala are critically involved in responses associated with fear, emotional responses, hormonal secretions and memory and their activity contribute to the receiving sensory information cerebral cortex that are highly involved in sensory perception and movement. Neuronal degeneration in these regions can cause a variety of sensory and motor illnesses, such as taste aversion [15], vomiting, headache, confusion, disorientation, seizures, myoclonus, coma [9] and in a severe condition lead to death [16]. Thalamus is also well positioned to relay sensory and motor signals to the cortex region [17]. Poisoning induced alteration may affect the thalamocortical connectivity, ultimately pays to the decline in motor and sensory functions such as maintaining posture, general movements and motor learning, vision, associated with $A$. hispidus poisoning [18]. As puffer fish poisoning damages spread through the brain, more regions become affected, the neocortex becomes damaged. Damages to the neocortex is linked to the problems with a decline in generation of motor commands and conscious thoughts [19]. Damage to the cerebellum in the brain makes precision, and accurate timing during poisoning condition [20], slurred speech, motor coordination, etc. [21].

Damages in the primary motor area, hippocampus CA2, CA3, amygdala, thalamus, and cerebellum could explain the problems with sensory and motor functions of the victims since the sensory and motor information processing centres are all located in this area. Quantitative analysis of regions suggests that these pathological alterations are dose depended. It is reasonable that to some extent the respiratory suffering and the cardiac failure that led death may have contributed to these morphological changes [22]. At the cellular level, neurons appeared to be more affected, and the neuron has considered as an important cellular target for the neurotoxic effects of TTX by altering action potential mechanism with significance neuronal death. Following exposure to poisoning condition, 
regions associated with sensory and motor functions are commonly known to effect TTX with the downregulation of the sodium ion channel [7]. Although we do not see the damages of the other regions of the brain, it's because of TTX was well evolved to bind the sodium channels of the neurons of precise areas of the brain for inhibition of sodium ions in those regions could effectively immobilize these tissues or led them to death [3].

The present study provides some valuable data's for the A. hispidus puffer fish poisoning evaluation. Results from the histological analysis support [9] that the A. hispidus poisoning is potent neurotoxins affecting the brain regions associated with sensory and motor functions. From our understanding that the changes described may be toxin-induced is supported by the pieces of evidence of the histopathological analysis and the changes appeared to be dose depended. Future studies will be the more detailed mapping of the site-specific neuronal injury on the rat brain intended for immunohistochemistry analysis.

\section{CONCLUSION}

The reports in the areas where puffer fishes are traditionally eaten is vital in ensuring peoples safety. To further enhance the security of the coastal colonizes, the investigation of fishes and elucidation of the neurotoxic effects in humans is imperative. However, due to lack of data's in human victims animal's models are following decent choice for analysing the neurotoxic effects puffer fish poisoning. It is particularly important since there is a global expansion of fish-eating culture and various puffer fish enjoyed as a delicacy in many countries. Puffer fish poisoning has not reported in India, but still, death or life-threatening conditions of the victims due to poisoning published in many countries. Hence, it is clear that the monitoring of puffer fish is necessary to ensure food safety. This current investigation demonstrated the value of histopathological analysis as a tool for analysing neurotoxicity. It is evident that further studies are needed and we hope of this preliminary study will stimulate the idea of research in this aspect.

\section{REFERENCE}

1. Abal, P., Louzao, M. C., Vilariño, N., Vieytes, M. R., \& Botana, L. M. (2019). Acute Toxicity Assessment: Macroscopic and Ultrastructural Effects in Mice Treated with Oral Tetrodotoxin. Toxins, 11(6), 305.

2. Noguchi, T., Onuki, K., \& Arakawa, O. (2011). Tetrodotoxin poisoning due to pufferfish and gastropods, and their intoxication mechanism. ISRN toxicology, 2011.

3. Yong, Y. S., Quek, L. S., Lim, E. K., \& Ngo, A. (2013). A case report of puffer fish poisoning in Singapore. Case reports in medicine, 2013.
4. Indumathi, S. M., \& Khora, S. S. (2017). Toxicity assessment and screening of tetrodotoxin in the oblong blowfish (Takifugu oblongus) from the Tamil Nadu Coast of Bay of Bengal, India. Asian Pacific journal of tropical medicine, 10(3), 278284.

5. Noguchi, T., Arakawa, O., \& Takatani, T. (2006). Toxicity of pufferfish Takifugu rubripes cultured in netcages at sea or aquaria on land. Comparative Biochemistry and Physiology Part D: Genomics and Proteomics, 1(1), 153-157.

6. Lago, J., Rodríguez, L. P., Blanco, L., Vieites, J. M., \& Cabado, A. G. (2015). Tetrodotoxin, an extremely potent marine neurotoxin: Distribution, toxicity, origin and therapeutical uses. Marine drugs, 13(10), 6384-6406.

7. Zimmer, T. (2010). Effects of tetrodotoxin on the mammalian cardiovascular system. Marine Drugs, 8(3), 741-762.

8. Fishbein, I., \& Segal, M. (2006). Miniature synaptic currents become neurotoxic to chronically silenced neurons. Cerebral Cortex, 17(6), 1292-1306.

9. Kiernan, M. C., Isbister, G. K., Lin, C. S. Y., Burke, D., \& Bostock, H. (2005). Acute tetrodotoxin-induced neurotoxicity after ingestion of puffer fish. Annals of neurology, 57(3), 339348.

10. Kerr, M. S., Sacré, P., Kahn, K., Park, H. J., Johnson, M., Lee, J., ... \& Liégeois-Chauvel, C. (2017). The role of associative cortices and hippocampus during movement perturbations. Frontiers in neural circuits, 11, 26.

11. Tan, Z. Y., Piekarz, A. D., Priest, B. T., Knopp, K. L., Krajewski, J. L., McDermott, J. S., ... \& Cummins, T. R. (2014). Tetrodotoxin-resistant sodium channels in sensory neurons generate slow resurgent currents that are enhanced by inflammatory mediators. Journal of Neuroscience, 34(21), 7190-7197.

12. Balleine, B. W., \& Killcross, S. (2006). Parallel incentive processing: an integrated view of amygdala function. Trends in neurosciences, 29(5), 272-279.

13. Buckner, R. L. (2013). The cerebellum and cognitive function: 25 years of insight from anatomy and neuroimaging. Neuron, 80(3), 807815.

14. Harrison, T. C., \& Murphy, T. H. (2013). Towards a circuit mechanism for movement tuning in motor cortex. Frontiers in neural circuits, 6, 127.

15. Roldan, G., \& Bures, J. (1994). Tetrodotoxin blockade of amygdala overlapping with poisoning impairs acquisition of conditioned taste aversion in rats. Behavioural brain research, 65(2), 213 219.

16. Vuilleumier, P., Chicherio, C., Assal, F., Schwartz, S., Slosman, D., \& Landis, T. (2001). Functional neuroanatomical correlates of 
hysterical sensorimotor loss. Brain, 124(6), 10771090.

17. Tyll, S., Budinger, E., \& Noesselt, T. (2011). Thalamic influences on multisensory integration. Communicative \& integrative biology, 4(4), 378381.

18. Miyachi, S., Lu, X., Inoue, S., Iwasaki, T., Koike, S., Nambu, A., \& Takada, M. (2005). Organization of multisynaptic inputs from prefrontal cortex to primary motor cortex as revealed by retrograde transneuronal transport of rabies virus. Journal of Neuroscience, 25(10), 2547-2556.

19. Dudman, J. T., \& Krakauer, J. W. (2016). The basal ganglia: from motor commands to the control of vigor. Current opinion in neurobiology, 37, 158-166.

20. Ackermann, H., Mathiak, K., \& Riecker, A. (2007). The contribution of the cerebellum to speech production and speech perception: clinical and functional imaging data. The Cerebellum, 6(3), 202-213.

21. Bane, V., Lehane, M., Dikshit, M., O'Riordan, A., \& Furey, A. (2014). Tetrodotoxin: Chemistry, toxicity, source, distribution and detection. Toxins, 6(2), 693-755.

22. Joob, B., \& Wiwanitkit, V. (2015). Puffer fish poisoning: Summary of case reports from Thailand. Journal of Coastal Life Medicine, 3(5), 416-417. 